\title{
Role of Oral Glutamine in Prevention and Treatment of Oral Mucositis in Head and Neck Cancer Patients Receiving Chemoradiation
}

\author{
Hashmath Khanum ${ }^{1}$, Iqbal Ahmed², V. Chendil², Rajesh Javarappa ${ }^{2}$ and Amrut Kadam ${ }^{2, *}$ \\ ${ }^{1}$ Radiation Oncology, Kidwai Cancer Institute, Bengaluru, India \\ ${ }^{2}$ Radiation Oncology, Bangalore Medical College \& Research Institute, Bengaluru, India
}

\begin{abstract}
Purpose: To evaluate the efficiency of glutamine in the prevention \& treatment of mucositis in head and neck cancer patients undergoing chemoradiation.

Material and Methods: Forty patients of histologically proven head and neck carcinomas undergoing chemoradiation with Conventional Radiation on telecobalt and concurrent Cisplatin were randomised into 2 groups. The study group received oral glutamine solution 2 hours prior to undergoing radiotherapy on all days of treatment. The severity and duration of mucositis were recorded once every week using WHO and RTOG grading system for all patients undergoing treatment.

Results: Glutamine lead to a delay in the onset of mucositis. The overall incidence of grade $\geq 3$ mucositis was significantly low in glutamine arm $(22 \%$ vs $55 \%, p=0.006)$. On weekly assessments, the incidence of grade $\geq 3$ mucositis in study arm compared to the control was 0 vs $30 \%, p=0.02$ at 4 weeks, 15.8 vs $45 \%, p=0.038$ at 5 weeks and 22 vs $70 \%, p=0.001$ at 6 weeks. However, there was no statistically significant difference in the incidence of grade 1 and 2 mucositis in both arms.

Conclusion: Use of oral glutamine reduces the incidence and duration of oral mucositis and hence helpful in the prevention and treatment of oral mucositis with good compliance and further result in good locoregional control of the disease.
\end{abstract}

Keywords: Chemoradiation, mucositis, head and neck cancers, glutamine.

\section{INTRODUCTION}

Head and neck cancers are the fourth most common cancers in India with an annual incidence of 1.3 million cases [1]. Radiotherapy is the cornerstone in the management of these cancers. However, it leads to unavoidable toxicities in the form of local reactions such as mucositis, loss of taste, decreased salivation and dysphagia [2].

Oral mucositis is the most common painful and dose-limiting side effect of therapeutic irradiation in locally advanced head and neck malignancies chemoradiation [3]. Severe inflammation \& injury to the oral mucosa can also increase the likelihood of oral or systemic infections [4]. Severe mucositis results in treatment interruptions, which can negatively influence prognosis [5].

Glutamine is the most abundant free amino acid in the body and shown to be the major metabolic fuel for intestinal tract [6]. Glutamine may help decrease mucous membrane injury induced by radiation by altering the inflammatory response. Glutathione, a byproduct of glutamine metabolism protects against oxidant injury [7].

*Address correspondence to this author Dr. Amrut Kadam at the Radiation Oncology, Bangalore Medical College \& Research Institute, Bengaluru, India; Tel: 9986545999; E-mail: raysoflife@gmail.com
Marked glutamine depletion develops over time in cancer patients causing cancer cachexia and compromise in acid-base balance, immune functions and epithelial integrity in the gut. This renders a negative impact on the function of host tissues dependent on glutamine stores for adequate functioning [8]. Hence the possible therapeutic role of glutamine in the prevention of host normal tissue toxicity during cancer treatment.

\section{AIMS AND OBJECTIVES}

The primary aim was to evaluate the safety and efficacy of glutamine in alleviating radiation and chemotherapy induced oral mucositis during chemoradiation of head and neck malignancies. The evaluation was based on a weekly comparison of RTOG acute morbidity scoring the grades of mucositis 1-4 of mucous membrane in head and neck patients of both arms. The secondary objective was the evaluation of locoregional control in the study population.

\section{MATERIALS AND METHODS}

A total of forty consecutive patients with histologically proven squamous cell carcinoma of head and neck being treated in the Department of Radiotherapy, Victoria Hospital were taken. Patients aged between 20 and 70 years with Karnofsky 
Table 1: Characteristics of the Study Population

\begin{tabular}{|c|c|c|c|}
\hline \multicolumn{2}{|c|}{ Parameters } & Test group & Control group \\
\hline \multicolumn{2}{|c|}{ Age (51-70yrs, $60 \%)$} & 12 & 12 \\
\hline \multirow[t]{2}{*}{ Gender } & Male & 18 & 12 \\
\hline & Female & 2 & 8 \\
\hline \multirow[t]{3}{*}{ Site } & Oral cavity & 8 & 13 \\
\hline & Oropharynx & 8 & 6 \\
\hline & Hypopharynx \& supraglottis & 4 & 1 \\
\hline
\end{tabular}

Performance status $\geq 70$ undergoing conventional radiotherapy with concurrent chemotherapy either radical or post-operative were included (Table 1). The patients with prior history of radiotherapy or chemotherapy or known seizure disorder or on any anticonvulsant, with distant metastasis or in whom treatment intent was palliative were excluded from the study. All patients will have normal baseline haematological, liver and renal profile.

After complete workup and evaluation, suitable patients with indication of concurrent chemoradiation were randomized to 2 arms with 20 patients in each arm. All patients were planned and treated on telecobalt for a dose of 60-70 Gy at 2Gy per fraction, 5 fractions per week over 6-7weeks, along with weekly chemotherapy with injection Cisplatin $40 \mathrm{mg} / \mathrm{m} 2$. The patients in study arm were given $10 \mathrm{gm}$ of glutamine in $1000 \mathrm{~mL}$ of water 2 hours prior to RT on all days of irradiation, the first few sips of which they had to swish and swallow.

All patients were assessed for oral mucositis and other acute toxicity symptoms using RTOG/ NCl-CTC acute morbidity grading once every week during the course of radiotherapy. The standard protocols for the maintenance of oral hygiene during radiotherapy were uniformly used for both the groups. Assessment was done at the completion of RT again including the primary lesion and the nodes involved were assessed separately as per the WHO Tumor Response Criteria. Patients were followed on a regular basis for a period of 3 months.

\section{RESULTS}

The data was analysed using two-tailed student ttest and Chi-square/ Fisher Exact test to find the significance of study parameters on a continuous and categorical scale between two groups. The mean dose of RT was $64 \mathrm{~Gy}$ with $78 \%$ of patients receiving 5 cycles of cisplatin and rest receiving at least 4 cycles. $90 \%$ of patients completed the entire treatment within an overall treatment time of 50 days.

The overall incidence of grade $\geq 3$ mucositis was significantly low in glutamine arm $(22 \%$ vs $55 \%, p=$ 0.006 ). On weekly evaluation, the incidence of grade $\geq$ 3 mucositis in study arm compared to the control was 0 vs $30 \%, p=0.02$ at 4 weeks, 15.8 vs $45 \%, p=0.038$ at 5 weeks and 22 vs $70 \%, p=0.001$ at 6 weeks. A similar trend continued post completion of RT with an earlier resolution of mucositis in the glutamine arm. All grade 3 mucositis resolved within 2 weeks post-RT in glutamine arm whereas $30 \%$ of grade $\geq 3$ mucositis in controls persisted up to 4 weeks ( 0 vs $30 \%, p=0.008$ ).

More than $97 \%$ of patients had a complete response in the primary, and $>92 \%$ had a complete nodal response.

\section{DISCUSSION}

Concurrent chemoradiation has been shown to improve survival and organ preservation in locally advanced head and neck cancers $[9,10]$ with an absolute survival benefit of $4.5 \%$ for chemoradiation in HNSCC [11]. Concurrent platinum-based chemotherapy regimens have demonstrated improved disease control rates compared to those using radiotherapy alone, but at the cost of increased highgrade mucositis, weight loss, hematologic and renal toxicity [12].

For a combination of chemoradiation regimen to be therapeutically beneficial, the ratio should be positive $(>1)$; that is, individual agents or their combination must be more effective against tumours than normal tissues [13]. Normal tissue sparing is to be a real challenge in multimodality treatment. 
Mucositis is an unavoidable acute morbidity of radiation while treating $\mathrm{HNC}$ patients. Mucositis is also a surrogate measure of the outcome because severe oral inflammation in such patients is strongly associated with an increased risk of sepsis, use of total parenteral nutrition, high hospital costs, and poor treatment results [4].

Glutamine is the most abundant amino acid. It is non-essential, but in instances of stress becomes conditionally essential and has to be supplemented [14]. In respect of tissue level glutamine plays an important role in maintaining the normal integrity of intestinal mucosa [15]. In patients with cancer, marked glutamine depletion develops over time causing cancer cachexia and compromise in acid-base balance, immune functions and epithelial integrity in the gut rendering a negative impact on the function of host tissues dependent on glutamine stores for optimal functioning.

Huang et al. [16] in a pilot randomized trial had demonstrated the influence of oral glutamine on radiation-induced oral mucositis in 17 patients of head and neck cancer receiving primary or adjuvant irradiation. Statistical significance was noted only in the analysis of objective mucositis $(p=0.0060)$ but not subjective mucositis $(p=0.1073)$. In the analysis of the

Table 2: Incidence of Grade $\geq 3$ Mucositis in both the Arms

\begin{tabular}{|c|c|c|c|c|}
\hline Mucositis (RTOG > =3 Grading) & Test Group (n=20) & Control Group $(\mathbf{n = 2 0 )}$ & Total (n=40) & P value \\
\hline \hline During RT (weeks) & $0(0 \%)$ & $6(30 \%)$ & $6(15 \%)$ & $0.020^{*}$ \\
\hline 4 & $3(15 \%)$ & $9(45 \%)$ & $12(30 \%)$ & $0.038^{*}$ \\
\hline 5 & $4(20 \%)$ & $14(70 \%)$ & $18(45 \%)$ & $0.001^{* *}$ \\
\hline 6 &
\end{tabular}

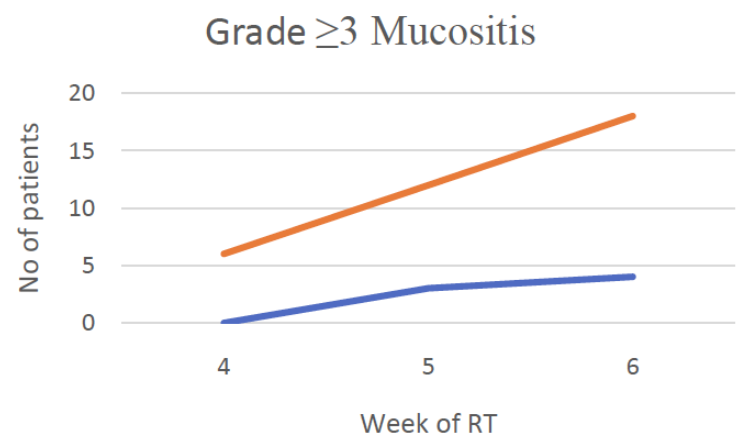

Test Group Control Group

a

\section{mucositis in test group}

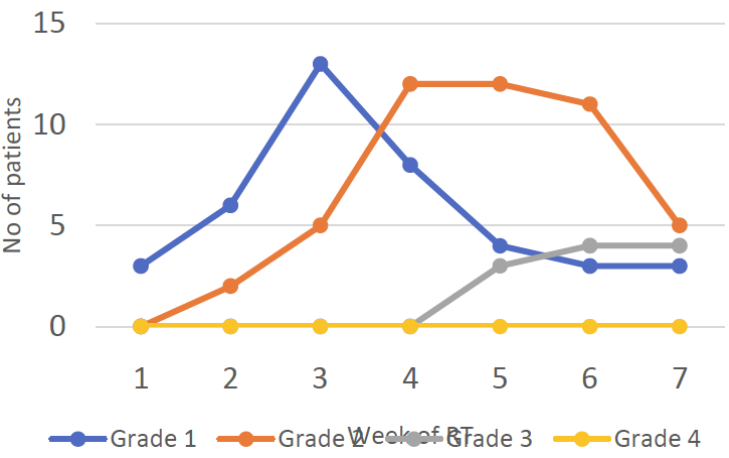

b

\section{mucositis in control group}

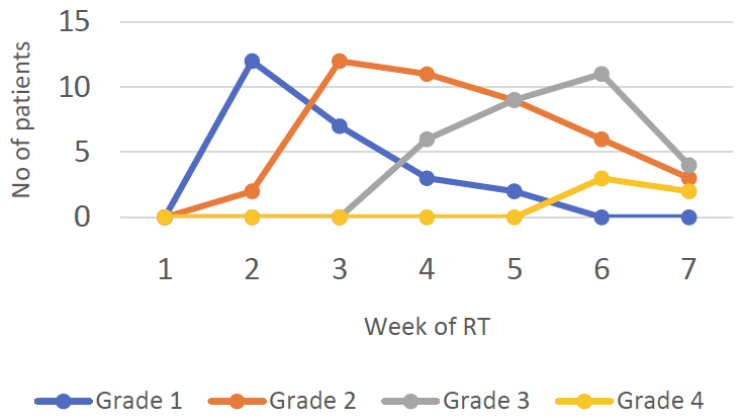

C

Figure 1: a: Incidence of grade $\geq 3$ mucositis. b: Incidence of mucositis in glutamine arm. $\mathbf{c}$ : Incidence of mucositis in controls. 
severity of mucositis, glutamine reduced the maximum grade of objective mucositis (mean 1.6 vs. 2.6) $(p=0.0058)$.

In our study, we found that there was no statistically significant difference in the incidence of grade 1 and 2 mucositis in both the arms, though the incidence of grade 2 and 3 mucositis was delayed by a week in the glutamine arm. The incidence of grade 3 mucositis with glutamine was $22 \%$ whereas in the controls it was $55 \%$ $(p=0.006)$. In addition, $15 \%$ of controls also had grade 4 mucositis which was not seen in study arm.

The incidence of grade $\geq 3$ mucositis on weekly assessment in the study and the control arm was 0 and $30 \%$ at 4 weeks $(p=0.02), 16 \%$ and $45 \%$ at 5 weeks $(p=0.038), 22 \%$ and $70 \%$ at 6 weeks $(p=0.001)$ (Table 2 ). The resolution of grade $\geq 3$ mucositis was complete within 2 weeks post-RT with glutamine, whereas $30 \%$ of controls still had progressively resolving mucositis $(p=0.02)$ (Table 2, Figure 1).

In a similar study by Subrata Chattopadhyay et al. [17], which evaluated the role of oral glutamine in a prospective randomized study involving 64 patients of head and neck cancer on radiation with or without concurrent chemotherapy, it was seen that the number of patients who developed grade 3 mucositis (14.29\%) and grade 4 mucositis $(2.86 \%$ ) in the study arm (who received oral glutamine) was significantly lower ( $p=$ 0.02 and $p=0.04$, respectively). The study showed that glutamine delays (mean time of onset) oral mucositis (all grades) and reduces the frequency and duration (mean duration) of grade 3 and 4 mucositis.

In another retrospective analysis by Alfonso VidalCasariego [18] use of glutamine was associated with a significant reduction in mucositis, weight loss and need of enteral nutrition.

In our study dysphagia as per RTOG grading was significantly lower in the study arm when compared to the control arm especially during the $2^{\text {nd }}$ and $4^{\text {th }}$ week of chemoradiation ( $p<0.001$ in both weeks). About $30 \%$ of patients in the glutamine arm maintained oral intake and did not require ryles tube feeding, while for the rest the requirement was delayed by 1 week when compared to controls.

This benefit of improvement in mucositis and better tolerance to treatment was noted without any compromise in the locoregional control in the immediate post RT period.

\section{CONCLUSION}

Oral glutamine use along with radiotherapy delays the onset and significantly reduces the incidence of severe oral mucositis in head and neck cancers treated with concurrent chemoradiation. There is a trend towards increasing the compliance to treatment while maintaining enteral nutrition and locoregional control. Glutamine supplementation will be beneficial to the patients in reducing the morbidity associated with oral mucositis.

However larger randomised studies with longer follow up duration are essential for further conclusions.

\section{REFERENCES}

[1] Takiar R, Nadayil D, Nandakumar A. Projections of Number of Cancer Cases in India (2010-2020) by Cancer Groups. Asian Pacific J Cancer Prev 11: 1045-1049.

[2] Zlotolow IM. General consideration in prevention and treatment of oral manifestation of cancer therapies. In: Berget AP, Weissman DE, editors. Principles and practice of surgical oncology. Philadelphia, PA: Lippincott raven; 1998, p. 237.

[3] Vissink A, Jansma J, Sp jkervet FK, Burlage FR, Coppes RP Oral sequelae of head and neck radiotherapy. Crit Rev Ora Biol Med 2003; 14:199-212. https://doi.org/10.1177/154411130301400305

[4] Trotti A, Bellm LA, Epstein JB, et al. Mucositis incidence, severity and associated outcomes in patients with head and neck cancer receiving radiotherapy with or without chemotherapy: a systematic literature review, Radiother Oncol 2003; 66: 253-262. https://doi.org/10.1016/S0167-8140(02)00404-8

[5] Mead GM. Management of oral mucositis associated with cancer chemotherapy. Lancet 2002; 359: 815-6. https://doi.org/10.1016/S0140-6736(02)07960-6

[6] Krebs H. Glutamine metabolism in animal body. In: Mora J Palacios R, editors. Glutamine: Metabolism, enzymology and regulation. New York: Academic press; 1980; pp. 319-25. https://doi.org/10.1016/B978-0-12-506040-0.50023-6

[7] Savarese DM, Savy G, Vahdat L, Wischmeyer PE, Corey B Prevention of chemotherapy and radiation toxicity with glutamine. Cancer Treat Rev 2003; 29: 501-13. https://doi.org/10.1016/S0305-7372(03)00133-6

[8] Klimberg VS, Souba WW, Dolson DJ, Salloum RM, Hautamaki RD, Plumley DA, et al. Prophylactic glutamine protects the intestinal mucosa from radiation injury. Cancer 1990; 66: 62-8 https://doi.org/10.1002/1097-0142(19900701)66:1<62::AIDCNCR2820660113>3.0.CO;2-E

[9] Vokes EE, Weichselbaum RR. Measurable impact: multimodality therapy of Head and neck cancer. Int J Radiat Oncol Biol Phys 1993; 27: 481-482. https://doi.org/10.1016/0360-3016(93)90263-U

[10] Forastiere AA, et al. Concurrent chemotherapy and Radiotherapy for organ preservation in advanced laryngeal cancer. N Engl J Med 2003; 349: 2091-2098. https://doi.org/10.1056/NEJMoa031317

[11] Jean-Pierre Pignon, Aurelie le Maitre a, Emilie Maillard, Meta-analysis of chemotherapy in head and neck cancer (MACH-NC): An update on 93 randomised trials and 17346 patients. Radiotherapy and Oncology 2009; 92: 4-14. https://doi.org/10.1016/j.radonc.2009.04.014 
[12] Blanchard P, Baujat B, Holostenco V. Meta-analysis of chemotherapy in head and neck cancer (MACH-NC): A comprehensive analysis by tumour site. Radiotherapy and Oncology 2011; 100: 33-40.

https://doi.org/10.1016/j.radonc.2011.05.036

[13] Principles and Practice of Radiation Oncology; 5th Edition: Carlos Perez, Luther Brady.

[14] Lacey, JM; Wilmore, DW. "Is glutamine a conditionally essential amino acid?" Nutrition Reviews 1990; 48(8): 297309.

https://doi.org/10.1111/j.1753-4887.1990.tb02967.x

[15] Yamamoto T, Shimoyama T, Kuriyama M (8 December). "Dietary and enteral interventions for Crohn's disease. Current Opinion in Biotechnology 2016; 44: 69-73. https://doi.org/10.1016/j.copbio.2016.11.011
[16] Huang EY, Leung SW, Wang CJ, Chen HC, Sun LM, Fang $\mathrm{FM}$, et al. Oral glutamine to alleviate radiation induced oral mucositis: A pilot randomized trial. Int J Radiat Oncol Biol Phy 2000; 46: 535-9.

https://doi.org/10.1016/S0360-3016(99)00402-2

[17] Chattopadhyay S, et al. Role of oral glutamine in alleviation and prevention of radiation induced oral mucositis: A prospective randomized study. South Asian Journal of Cancer 2014; 3(1): 8-12. https://doi.org/10.4103/2278-330X.126501

[18] Vidal-Casariego A, et al. Efficacy of glutamine in prevention of oral mucositis and radiation induced esophagitis: retrospective study. Nutrition and Cancer 2013; 65(3): 424429.

https://doi.org/10.1080/01635581.2013.765017

Received on 20-04-2019

Accepted on 15-05-2019

Published on 08-07-2019

https://doi.org/10.30683/1927-7229.2019.08.02

(C) 2019 Khanum et al.; Licensee Neoplasia Research.

This is an open access article licensed under the terms of the Creative Commons Attribution Non-Commercial License (http://creativecommons.org/licenses/by-nc/3.0/) which permits unrestricted, non-commercial use, distribution and reproduction in any medium, provided the work is properly cited. 